BULLETIN Bulletin hispanique

HISPANIQUE Université Michel de Montaigne Bordeaux

110-1 | 2008

Varia

\title{
Luis Díaz Viana, Cancionero popular de la Guerra civil española. Textos y melodías de los dos bandos
}

\author{
J.-F. Botrel
}

\section{(2) OpenEdition}

Journals

Édition électronique

URL : https://journals.openedition.org/bulletinhispanique/702

DOI : 10.4000/bulletinhispanique.702

ISSN : 1775-3821

Éditeur

Presses universitaires de Bordeaux

Édition imprimée

Date de publication : 1 juin 2008

Pagination : 391-393

ISBN : 978-2-86781-511-9

ISSN : 0007-4640

Référence électronique

J.-F. Botrel, « Luis Díaz Viana, Cancionero popular de la Guerra civil española. Textos y melodías de los dos bandos », Bulletin hispanique [En ligne], 110-1 | 2008, mis en ligne le 10 février 2014, consulté le 18

septembre 2021. URL : http://journals.openedition.org/bulletinhispanique/702 ; DOI : https://doi.org/ 10.4000/bulletinhispanique.702

Tous droits réservés 
Daubenton escrita por su compañero, el Padre Frizon. Con ella, ilumina dos rincones oscuros, y sin embargo decisivos, de la maquinaria que regía los estados modernos: el proceso de toma de decisión en el nivel más alto; el punto de engarce de lo religioso y de lo civil, el punto desde el cual los rectores de la sociedad religiosa transmitían su mensaje al rector supremo de la sociedad civil.

Como era de esperar en un trabajo de principiante, el libro tiene su lado débil. España casi no aparece. La acción de Daubenton en Madrid se enfoca exclusivamente en relación con la problemática de las luchas internas al bando francés en las cortes de Madrid y de París. No se dice nada de la administración del patronato real y de la función de regulación interna de la iglesia de España, que eran también tareas fundamentales encargadas al confesor. La documentación manejada es casi exclusivamente francesa, las obras de Poutrin y Alcaraz ni se citan en la bibliografía. La problemática general es cuando menos floja, y muy por debajo de lo que exigen los datos proporcionados: rehabilitar la persona del protagonista es un objetivo simpático pero acientífico. Se pueden pasar por alto tales insuficiencias en nombre de la aportación del libro y de la juventud del autor. Tendrán sin embargo que quedar subsanadas en la tesis que Mlle Desos está preparando sobre el «entourage » francés de Felipe V en Madrid, tema en el que enfrentará un nivel de exigencias más alto y la dura competencia de los trabajos recientes de Concepción de Castro, Anne Dubet y Thomas Glesener. ¡A ver!

\section{Jean-Pierre DEDIEU}

Luis Díaz Viana, Cancionero popular de la Guerra civil española. Textos y melodias de los dos bandos. Prólogo de José-Carlos MaIner, Madrid, La Esfera de los Libros, 2007, 301 p. - ISBN 8497346408. - ISBN-13 9788497346405.

Il s'agit de la réédition d'un livre publié en 1985 à partir de recherches menées par l'anthropologue du C.S.I.C. à la fin des années 1970, sur cette " autre littérature " qu'est la chanson, à une époque où, à propos de la littérature orale et populaire de la Guerre civile, on ne connaissait guère que le Romancero de la guerra de España de Serge Salaün.

Le risque existe de voir une initiative et une étude pionnières - "corrigée et augmentée ", comme on disait, mais sans actualisation de la bibliographie - réinvestie sur le mode de la nostalgie. Cependant, en plein débat sur la mémoire historique, une nouvelle lecture de cette étude, opportunément resituée, à travers un Préambule (pp. 19-35) et une réflexion 
sur l'objet de l'étude une fois celle-ci achevée, dite «Epígono » (pp. 271286), dans l'histoire des recherches sur la Guerre civile et la littérature orale est d'une étonnante actualité.

D'abord parce qu'elle permet de rappeler, à propos de chansons "populaires ", c'est-à-dire, pour suivre la définition de Díaz Viana (p. 301), celles qui sont « connues et partagées à différents moments par beaucoup de gens » et qui sont ici évidemment liées à l'action, que, au delà des origines plus ou moins traditionnelles des mélodies ou des paroles, l'ensemble des procédés et mécanismes de création littéraire mis en œuvre sont fortement articulés à la performance. Car l'oralité littéraire, qu'il s'agisse d'une oralité primaire, mixte ou médiatisée, pour reprendre les catégories de Zumthor, est un phénomène plus ample et moins évanescent que ce qu'on appelle la tradition orale et tout un pan de la culture du peuple - en l'occurrence, la chanson - reste encore largement à considérer, y compris en dehors des contextes de conflits aigus.

Le préambule à cette nouvelle édition, intitulé " Canciones como balas o el retumbar de un tiempo mítico ", est l'occasion pour L. Díaz Viana de mettre en perspective - scientifiquement et vitalement - cette recherche pionnière en son temps, qui n'est pas une méditation à la Vázquez Montalbán, mais toujours un ouvrage positif qui fournit pour chacun des camps - le « bando republicano " (pp. 57-149) et le « bando nacional » également dit « de las fuerzas sublevadas" (pp. 151-269) - la transcription des textes recueillis à partir de sources écrites et d'informations orales ainsi que celle de la musique qui, pour les analphabètes de la partition, aurait bien mérité d'être enregistrée sur un $\mathrm{CD}$-, mais aussi de systématiques et savantes considérations sur les origines et l'évolution des chansons recueillies, des plus connues, comme "Si me quieres escribir " ou "Cara al sol ", jusqu'à "En pos de la vida " (sur une musique de Chostakovich) ou "Los Flechas ». C'est l'occasion d'observer que ce qui peut être considéré comme un folkore agglutinant de deux ensembles, comporte des nuances internes et se définit de façon assez contrastée par rapport à la métrique qui renvoie, dans le premier cas, à des modèles considérés comme populaires et, dans le second, à des prétentions cultistas ou à certain goût pour une artificiosité qu'on assimile souvent au " littéraire" (p. 283).

Au delà, ce " cancionero de la guerra » est, comme le rappelle justement José Carlos Mainer dans son prologue (p. 17), « un buen pretexto para meditar lo cerca que se andan el heroísmo y la crueldad, la exaltación gregaria y la entrega más noble a una causa, el machismo más innoble y la ternura más húmeda ». C'est aussi - en permanence - une stimulante invitation à se demander, avec Díaz Viana, pour la science et pour soi - la chanson étant 
particulièrement utile à cet égard, car comme le dit l'auteur (p. 34) «las canciones casi siempre tiran de otros recuerdos»-, non pas comment "fue el pasado en el pasado ", mais comment " el pasado se continúa o perpetúa en el presente ".

J.-F. BOTREL

Milagros Ezquerro, Leerescribir. - Mexico-Paris, Rilma-ADEHL, 2008, 197 p. - ISBN 978-970-95635-1-1.

La professeure Milagros Ezquerro présente avec Leerescribirl'aboutissement de nombreuses années de recherche, toute une carrière consacrée à l'analyse littéraire et à la théorie de la littérature. Milagros Ezquerro est une éminence de l'américanisme français, fidèle à l'analyse d'un corpus textuel qui ne cesse de s'accroître.

Leerescribirvient de sortir, édité à Mexico, en partenariat avec les universités de Limoges et de la Sorbonne. Il s'agit du $5^{\mathrm{e}}$ titre de la collection Estudios y ensayos qu'animent Milagros Ezquerro et le professeur Eduardo RamosIzquierdo. Leerescribir est un mot-valise, autrement dit un néologisme. Ce qu'entend démontrer Milagros Ezquerro, et qu'annonce le titre de cet essai, c'est que lire et écrire sont deux actes qui se télescopent en permanence. L'un n'a pas de sens sans l'autre; l'un est l'autre.

Dans cette démonstration, Milagros Ezquerro rappelle que le texte littéraire est un système complexe qui fonctionne en relation avec deux autres systèmes tout aussi complexes quoique cette complexité soit imperceptible au lecteur lambda. L'approche de Milagros Ezquerro rappelle celle de la physique nucléaire. Mais sa démonstration n'exige pas de connaître une infinité de théorèmes. Pour les scientifiques de la matière, l'atome est la plus petite partie d'un corps simple ; il est généralement constitué d'un noyau autour duquel gravitent des électrons. Dans Leerescribir, Milagros Ezquerro définit d'une part le producteur du texte, qu'elle appelle le Sujet Alpha et l'observateur du texte, le Sujet Omega. Ces deux Sujets aux identités plurielles gravitent et échangent mutuellement avec l'objet qui est au centre de tout : le texte littéraire.

Le sujet Alpha ne se confond pas avec l'auteur, qui écrit le texte. C'est l'auteur mais aussi tout son environnement, tout ce qui a participé au processus de création, la culture de l'auteur, sa vie privée ou publique, les agents secondaires de la production, qu'il s'agisse des maîtres à penser ou des disciples, de l'éditeur qui impose un titre ou de l'illustrateur. Le concept de Sujet Alpha est donc novateur; il s'efforce d'embrasser la totalité du 